\title{
Changing demographics of pulmonary arterial hypertension in congenital heart disease
}

\author{
B.J.M. Mulder
}

ABSTRACT: Pulmonary arterial hypertension (PAH) is a serious complication of congenital heart disease (CHD). Without early surgical repair, around one-third of paediatric $\mathrm{CHD}$ patients develop significant PAH. Recent data from the Netherlands suggest that $>4 \%$ of adult $\mathrm{CHD}$ patients have PAH, with higher rates in those with septal defects. A spectrum of cardiac defects is associated with PAH-CHD, although most cases develop as a consequence of large systemic-to-pulmonary shunts. Eisenmenger's syndrome, characterised by reversed pulmonary-to-systemic (right-to-left) shunt, represents the most advanced form of PAH-CHD and affects as many as $50 \%$ of those with PAH and left-to-right shunts. It is associated with the poorest outcome among patients with PAHCHD. 40 yrs ago, $\sim 50 \%$ of children with $\mathrm{CHD}$ requiring intervention died within the first year, and $<15 \%$ survived to adulthood. Subsequent advances in paediatric cardiology have seen most patients with CHD survive to adulthood, with resulting shifts in the demographics of CHD and PAH-CHD. The number of adults presenting with CHD is increasing and, although mortality is decreasing, morbidity is increasing as older patients are at increased risk of arrhythmia, heart failure, valve regurgitation and PAH. Data show that probability of PAH increases with age in patients with cardiac defects.

KEYWORDS: Congenital heart disease, pulmonary arterial hypertension

$\mathbf{P}$ ulmonary arterial hypertension (PAH), a disease of the pulmonary vasculature characterised by an elevated mean pulmonary arterial pressure $\left(\bar{P}_{\text {pa }}\right)$ at rest of $\geqslant 25 \mathrm{mmHg}[1]$, is one of the most serious complications of congenital heart disease (CHD). After idiopathic PAH (IPAH) and PAH associated with connective tissue diseases (PAH-CTD), it is the third most common form of PAH [2].

A diagnosis of $\mathrm{PAH}$ has ramifications for both paediatric and adult CHD patients. In children, the course of CHD is complicated by the presence of PAH: estimates suggest that approximately one-third of paediatric CHD patients develop significant PAH without early surgical repair [3]. Increasing numbers of adults are presenting with $\mathrm{CHD}$, illustrated by the fact that there are 1.2 million adult CHD patients in Europe alone, all of whom are at risk of developing $\mathrm{PAH}$. The prevalence of PAH associated with CHD (PAHCHD) was calculated as $4.2 \%$ among a population of 5,970 CHD patients included in the Dutch
CONCOR registry, rising to $6.2 \%$ among 1,824 patients with septal defects [4]. In a separate Dutch study, $9.9 \%$ of 1,148 patients with CTD were found to have PAH [5].

PAH can occur in association with a number of conditions other than CHD, although the underlying pathophysiology is similar in each case. Previous studies have suggested that PAH-CHD has a better prognosis than PAH of other aetiologies. Research has demonstrated an actuarial 3-yr survival rate for PAH-CHD of $77 \%$, compared with only $35 \%$ at 3 yrs for untreated IPAH [6], $37 \%$ for PAH-CTD and $21 \%$ for PAH associated with HIV infection [3].

Although progression of PAH appears slower in patients with CHD compared with IPAH and $\mathrm{PAH}$ of other aetiologies, it remains an extremely debilitating condition that affects both survival and quality of life [7]. Moreover, because greater numbers of patients with CHD now survive into adulthood, the demographics of PAH-CHD are changing. This article describes the wide range of
CORRESPONDENCE B.J.M. Mulder Dept of Cardiology, B2-240 Academic Medical Centre Meibergdreef 9 1105AZ Amsterdam The Netherlands E-mail: b.j.mulder@amc.uva.nl

Received: Aug 242010 Accepted after revision: Sept 102010

\section{PROVENANCE}

Publication of this peer-reviewed article was supported by Actelion Pharmaceuticals Ltd, Switzerland (unrestricted grant, European Respiratory Review issue 118). 
cardiac defects that are associated with the development of $\mathrm{PAH}$ in $\mathrm{CHD}$, and discusses the changing demographics of PAH-CHD.

\section{CLINICAL PRESENTATION OF PAH-CHD}

In patients with CHD, the development of $\mathrm{PAH}$ has been associated with a broad spectrum of congenital cardiac lesions (table 1), which can be divided into four categories [8]. The first category includes heart lesions that cause left-to-right shunts, resulting in blood flow through the pulmonary vasculature that is elevated in terms of both rate and pressure; such defects include atrial septal defect (ASD), ventricular septal defect (VSD), atrioventricular septal canal defects and patent ductus arteriosus (PDA). The second category comprises lesions such as mitral stenosis and aortic stenosis, which cause left-sided (pulmonary venous) obstruction. The third category consists of a subgroup of cyanotic heart lesions when they present with increased pulmonary blood flow, such as common arterial trunk and transposition of the great vessels. The final category includes anomalies of the pulmonary arteries, such as hemitruncus and tetralogy of Fallot. It is important to note that the latter causes PAH only when it occurs simultaneous with pulmonary atresia and major aortopulmonary collateral artery, as tetralogy of Fallot alone obstructs the pulmonary blood flow and prevents overcirculation in the pulmonary arteries [8].

Most cases of PAH develop as a consequence of large systemicto-pulmonary shunts, where exposure of the pulmonary vasculature to high systemic arterial pressure leads to increased pulmonary arterial pressure $(P$ pa $)$, progressive pulmonary vascular injury and increased pulmonary vascular resistance (PVR) [9]. As mentioned previously, data from the CONCOR registry of adult, Dutch CHD patients provide insights into the frequency of $\mathrm{PAH}$ in relation to various cardiac defects. Among patients with ASD, some 7-8\% developed PAH, compared with $11 \%$ of those with VSD and $41 \%$ of those with atrioventricular septal canal defects [4]. An earlier, retrospective comparison between patients with ostium secundum ASD, when the aperture in the second septum of the fetal heart fails to close, and those with sinus venosus defects, where the superior portion of the atrium fails to develop, showed that $P$ pa and PVR were higher in patients with sinus venosus defects. Not surprisingly, the prevalence of PAH was also higher in these patients ( $26 \%$ versus $9 \%$, respectively) [10].

In cases where changes in the pulmonary arteries (irreversible vascular injury) cause PVR to exceed systemic vascular resistance, a reversed pulmonary-to-systemic (right-to-left) shunt can occur [1]. Development of this condition, known as Eisenmenger's syndrome, represents the most severe form of PAH-CHD. Surveys suggest that Eisenmenger's syndrome affects up to $1 \%$ of adult CHD patients and $6 \%$ of those with shunts [4]. Eisenmenger's syndrome includes all systemic-topulmonary shunts that arise from large septal defects and that ultimately lead to pulmonary-to-systemic or bidirectional shunts, with associated symptoms of cyanosis, erythrocytosis and multiple organ involvement.

Patients with CHD who develop Eisenmenger's syndrome have a variety of congenital heart defects, of which VSD, ASD and PDA are among the most common. Recent data from the CONCOR registry showed that, among patients with $\mathrm{PAH}$ and septal defects, more than one-half $(58 \%)$ had Eisenmenger's syndrome, representing $\sim 1 \%$ of the total patient population. It was more than twice as common among patients with VSD (48\%) relative to those with ASD (17\%) [4], consistent with earlier reports. For example, in a long-term observational cohort study of 1,280 patients with VSD, most of whom were infants at study entry, 98 (7.8\%) were diagnosed with Eisenmenger's syndrome on admission [11]. Eisenmenger's syndrome subsequently developed in 10 of the 322 patients who were managed medically, and in one of the 226 patients who underwent surgical closure of the defect. Among the medically managed patients, there were no cases of Eisenmenger's syndrome in patients with trivial or mild VSDs, two $(3.1 \%)$ cases in those with moderate VSDs and eight $(57.1 \%)$ cases in those with severe VSDs [11].

Other data also indicate that Eisenmenger's syndrome is more likely to develop in patients with large and complex septal defects. For example, data have shown that Eisenmenger's syndrome develops in $10 \%$ of patients with unrepaired large ASD, in $50 \%$ of patients with unrepaired large VSD or PDA and in almost all patients with unrepaired common arterial trunk [12]. Interestingly, onset of Eisenmenger's syndrome

TABLE 1 Pulmonary arterial hypertension associated with congenital heart disease (CHD)

\begin{tabular}{ll} 
Type of defect & Example \\
\hline Left-to-right shunts & Atrial septal defect \\
& Ventricular septal defect \\
& Atrioventricular defects \\
Left-sided obstruction & Patent ductus arteriosus \\
Cyanotic CHD (occurring with increased pulmonary blood flow) & Mitral stenosis \\
& Aortic stenosis \\
Anomalies of pulmonary arteries & Common arterial trunk \\
& Transposition of great vessels \\
& Tetralogy of Fallot with pulmonary atresia and MAPCAs
\end{tabular}

MAPCA: major aortopulmonary collateral artery. Modified from [8] with permission from the publisher. 
tends to be early in patients with VSD or PDA, among whom $80 \%$ of cases occur during infancy, whereas among patients with ASD, 90\% of cases occur during adulthood [12].

As these data illustrate, the risk of developing irreversible pulmonary vascular disease depends on the specific nature and size of the cardiac lesion, as well as the degree of pulmonary hypercirculation, $P$ pa and the level of hypoxia. Among patients with PAH-CHD, those with Eisenmenger's syndrome tend to have the poorest prognosis. Data from a large case-control study of adult CHD patients matched with healthy individuals showed that median survival was greatly reduced in Eisenmenger's patients (fig. 1). Of the cohort of 171 CHD patients, 97 (57\%) had ASD, VSD or PDA, while $74(44 \%)$ had complex cardiac defects that included univentricular heart, transposition of the great arteries and common arterial trunk [13]. Eisenmenger's patients with complex cardiac defects were at increased risk of mortality compared with those with simple lesions. As figure 1 illustrates, the presence of complex lesions in Eisenmenger's syndrome patients was associated with a survival reduction of $20 \mathrm{yrs}$, compared with patients with simple lesions; relative to subjects without Eisenmenger's syndrome, this represents a 40-yr reduction in survival. Poor World Health Organization functional class (WHO FC), clinical signs of heart failure and a history of clinical arrhythmia were also predictive of increased mortality in these patients [13].

Patients with PAH-CHD are generally considered to have a more positive prognosis relative to those with other forms of PAH (fig. 2) [3]. However, data from REVEAL, a multicentre, observational, US-based registry study of $\mathrm{PAH}$, show that, in contrast to historical data, patients with PAH-CHD today have similar 1- and 5-yr outcomes to those with IPAH [14]. This apparent change in outcome may be attributable to the availability of new, targeted treatments for $\mathrm{PAH}$ and their use in combination therapy, which has improved prognoses for patients with IPAH. More recently, studies on the use of targeted PAH therapies in PAH-CHD, which have included patients with Eisenmenger's syndrome, have suggested that such targeted therapies may also improve the natural history of the disease in patients with PAH-CHD [15-19].

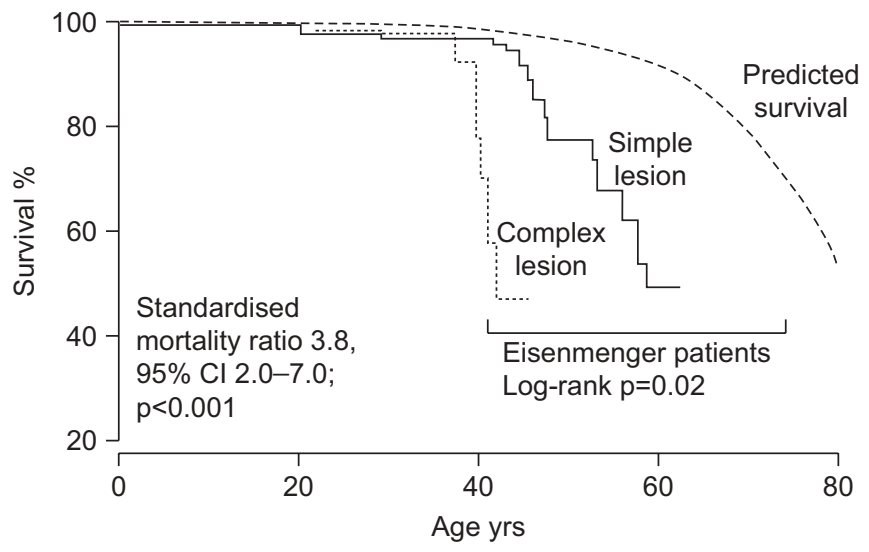

FIGURE 1. Survival prospects of patients with Eisenmenger's syndrome in comparison with an age- and sex-matched healthy population. Reproduced from [13] with permission from the publisher.

\section{CHANGING DEMOGRAPHICS OF CHD}

Before 1970, $\sim 50 \%$ of children with CHD requiring intervention died within the first year, and $<15 \%$ survived to adulthood [20]. Major advances in paediatric cardiology and surgery over the past 40 yrs have seen an increasing number of patients with CHD survive to adulthood [21]. Nowadays, $>85 \%$ of infants with CHD can be expected to reach adulthood [21]. This has shifted the demographics in developed countries, so that there are now more adults than children with CHD. Adult CHD patients consist of those who underwent surgery during infancy or childhood, some of whom may have been lost to follow-up after repair, and those in whom CHD went unrecognised until later in life.

This changing demographic picture can be observed in data from a Canadian population-based study of the prevalence and age distribution of CHD in the general population between 1985 and 2000 (fig. 3) [22]. As figure 3 shows, no change was observed in the prevalence of CHD in children between 1985 and 2000, but many more patients survived into adulthood in 2000. In fact, there was an $85 \%$ increase in adult prevalence between 1985 and 2000, with the largest increase occurring in adolescents and young adults. This pattern is consistent with data on the age distribution of CHD from the Mayo Clinic, USA, which followed adult patients transferred from an existing paediatric programme [23], and from the CONCOR registry in the Netherlands [24]. In both cases, prevalence of CHD was highest in patients aged 20-30 yrs, reflecting improvements in diagnosis, surgery and postoperative care, while $75 \%$ of cases occurred in patients aged $<50$ yrs $[23,24]$.

As the number of adult patients with CHD has increased, a new set of challenges has arisen. For instance, adult CHD patients may have to undergo repeated operations. In addition, technical advances have enabled the repair of more complex heart defects that were once inoperable, with operations becoming more intricate as a consequence. Finally, the number of percutaneous interventions is increasing. Thus, while mortality from CHD is decreasing, morbidity is increasing, especially in older cohorts of patients. Data from the Euro Heart Survey on 4,110 adult patients diagnosed with CHD and followed-up over a 5-yr period showed that, while mortality was relatively low over the follow-up period $(2.8 \%)$, almost one-quarter of patients had a history of major morbidity at baseline. Arrhythmias (23\%), stroke/transient ischaemic attack $(4 \%)$ and endocarditis (3\%) were among the most common adverse events reported [25]. A similar pattern was seen in the data from the CONCOR registry, in which longer term complications of CHD included increased risk of arrhythmia, heart failure (often of the right ventricle), valve regurgitation and PAH [24]. As the population of patients with CHD grows and ages, so the number of patients presenting with these complications is likely to increase.

\section{CHANGING DEMOGRAPHICS OF PAH-CHD}

In parallel with changes in the demographics of CHD, there have been corresponding shifts in the demographics of $\mathrm{PAH}-$ CHD. These changes are toward not only more adult patients [26], but also fewer adult CHD patients with PAH and simple cardiac lesions and increased numbers of patients with more complex lesions and PAH [7, 22]. 


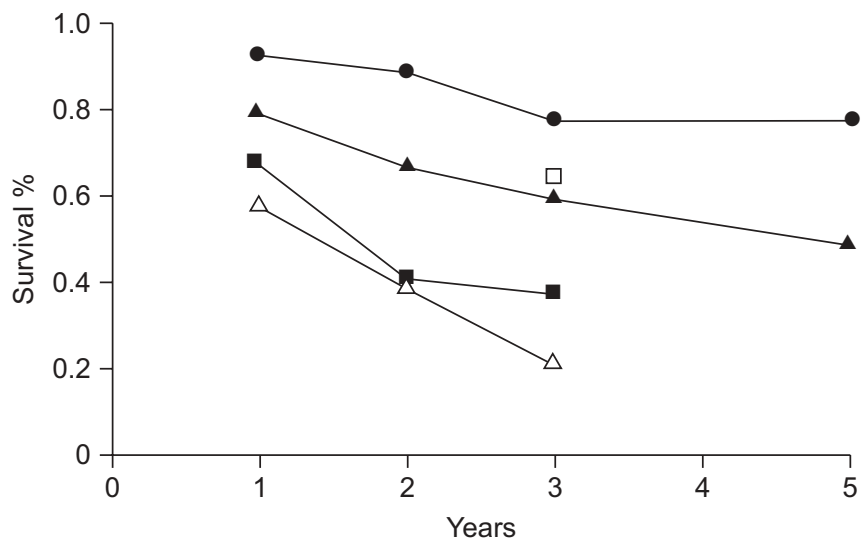

FIGURE 2. Survival in patients with pulmonary arterial hypertension based on aetiology. - congenital heart disease; $\mathbf{\square}$ : collagen vascular disease; $\triangle$ : HIVrelated; $\mathbf{\Lambda}$ : idiopathic pulmonary arterial hypertension; $\square$ : portopulmonary hypertension. Reproduced from [3] with permission from the publisher.

While the short-term prognosis of PAH-CHD is often good, PAH-CHD patients do not experience stable disease, and those in the fifth and sixth decades of life exhibit a trend toward increased morbidity and mortality. Data from the Euro Heart Survey on the prevalence of PAH among 1,877 adult CHD patients with septal defects have shown that the probability of PAH increases with age [27]. Of the total population, selected from referral centres based on a diagnosis of ASD, VSD, Eisenmenger's syndrome or another cyanotic defect, $28 \%$ of patients had PAH. Prevalence was higher in patients with open VSD and ASD (28 and 34\%, respectively), compared with those with closed defects (12 and 13\%, respectively). Increases in systolic $P$ pa were associated with increased functional impairment in patients with both ASD and VSD (fig. 4), while increased systolic Ppa was also associated with increased prevalence of right ventricular dysfunction among patients with unclosed ASD. Of the 1,877 patients, $231(12.3 \%)$ had Eisenmenger's syndrome. These patients had the highest rates of functional impairment and mortality; $20.6 \%$ of Eisenmenger's patients died over the 5-yr follow-up period. In patients with an open defect, PAH led to an eight-fold increase in functional impairment (functional impairment defined as being in WHO

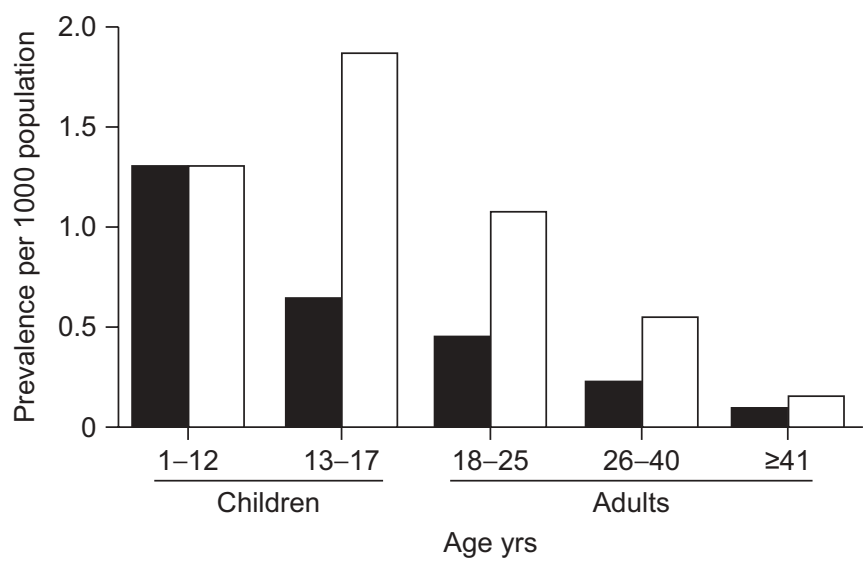

FIGURE 3. Changing prevalence of congenital heart disease between 1985 (Ш) and $2000(\square)$ in the Canadian general population. Reproduced from [22] with permission from the publisher.

FC II or above), with a further six-fold increase in patients with Eisenmenger's syndrome [27].

Given the inherent prognostic significance for $\mathrm{CHD}$ patients of a diagnosis of $\mathrm{PAH}$, it is important to screen CHD patients who are at risk of developing the condition, especially since effective treatments for Eisenmenger's syndrome have become available. The European Respiratory Society/European Society of Cardiology guidelines recommend treating patients with Eisenmenger's syndrome who are in WHO FC III with bosentan (recommendation class 1, level of evidence B) or other endothelin-receptor antagonists, phosphodiesterase type- 5 inhibitors or prostanoids (class of recommendation IIa, level of evidence C) [1].

Down's syndrome represents another patient population at risk for PAH-CTD. Individuals with Down's syndrome are often born with heart defects: a prospective study by the Dutch Paediatric Surveillance Unit in children with Down's syndrome, born between 2003 and 2006, showed that $43 \%$ of 482 children in the cohort had CHDs [28]. The most common defect was ASD, which occurred in $54 \%$ of patients, with VSD occurring in $33.3 \%$ and PDA in 5.8\% [28]. In patients with Down's syndrome, it has
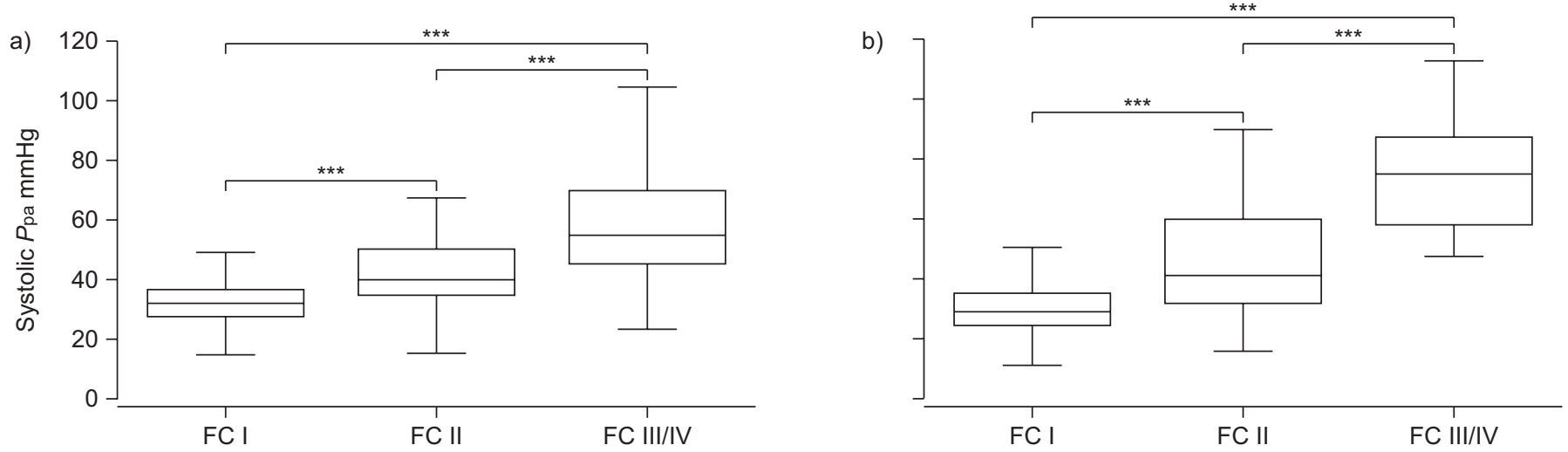

FIGURE 4. Distribution of systolic pulmonary artery pressure $(P$ pa) in relation to functional class (FC) for congenital heart disease patients with a) atrial septal defect and b) ventricular septal defect, but without Eisenmenger's syndrome. ${ }^{* *}$ : $p<0.001$. Reproduced from [27] with permission from the publisher. 
been suggested that PAH-CHD develops earlier and more aggressively [29, 30]. In the Belgian national registry of Eisenmenger's syndrome patients, $45 \%$ of the 91 patients included had Down's syndrome [31]. Patients with dual Down's-Eisenmenger's syndrome were younger than those with Eisenmenger's syndrome and had a worse functional capacity. They were also less likely to be receiving advanced PAH therapy. Bosentan has been investigated for its effects in patients with both Eisenmenger's syndrome and Down's syndrome [18]: median 6-min walk distance (6MWD) significantly increased among bosentan-treated patients over 12 weeks, but not over 52 weeks. The results were consistent with those observed in Eisenmenger's syndrome patients without Down's syndrome, and it appears that bosentan may be of some benefit in dual Down's-Eisenmenger's syndrome patients, especially in the short term. However, it is questionable whether 6MWD is a reliable measure in Down's syndrome patients [32].

\section{CONCLUSIONS}

Despite major advances in paediatric cardiology over the past 40 yrs, PAH remains a serious complication in CHD patients, and $\mathrm{PAH}-\mathrm{CHD}$ is the third most common form of PAH after IPAH and PAH-CTD.

Today, the majority of patients with PAH-CHD are adults, a situation that reflects the changing demographics of $\mathrm{CHD}$, wherein many more children with CHD survive to adulthood. While the development of PAH is associated with a wide variety of cardiac defects, most cases of PAH develop as a consequence of large systemic-to-pulmonary shunts that can lead to Eisenmenger's syndrome, especially in patients with large, complex cardiac lesions. Within the PAH-CHD population, patients with Eisenmenger's syndrome have the highest rates of functional impairment and mortality. Although we do not know how the prevalence of PAH will change in the next few years, it is likely that the number of cases of Eisenmenger's syndrome will decrease, while the number of PAH cases in CHD patients with complex heart defects will increase. The demographic shift toward a more adult population of patients with PAH-CHD, many with increasingly complex cardiac defects, presents a major therapeutic challenge.

\section{STATEMENT OF INTEREST}

None declared.

\section{ACKNOWLEDGEMENTS}

Editorial assistance was provided by L. Thomas (Elements Communications Ltd, Westerham, UK), supported by Actelion Pharmaceuticals Ltd (Allschwil, Switzerland).

\section{REFERENCES}

1 Galiè N, Hoeper MM, Humbert $M$, et al. Guidelines for the diagnosis and treatment of pulmonary hypertension: The Task Force for the Diagnosis and Treatment of Pulmonary Hypertension of the European Society of Cardiology (ESC) and the European Respiratory Society (ERS), endorsed by the International Society of Heart and Lung Transplantation (ISHLT). Eur Heart J 2009; 30: 2493-2537.

2 Hoeper MM. Definition, classification, and epidemiology of pulmonary arterial hypertension. Semin Respir Crit Care Med 2009; 30: 369-375.
3 McLaughlin VV, Preaberg KW, Doyle RL, et al. Prognosis of pulmonary arterial hypertension: ACCP evidence-based clinical practice guidelines. Chest 2004; 126: Suppl. 1, 78S-92S.

4 Duffels MGJ, Engelfriet PM, Berger RMF, et al. Pulmonary arterial hypertension in congenital heart disease: an epidemiologic perspective from a Dutch registry. Int J Cardiol 2007; 120: 198-204.

5 Vonk MC, Broers B, Heijdra YF, et al. Systemic sclerosis and its pulmonary complications in the Netherlands: an epidemiological study. Ann Rheum Dis 2009; 68: 961-965.

6 Hopkins WE, Ochoa LL, Richardson GW, et al. Comparison of the hemodynamics and survival of adults with severe primary pulmonary hypertension or Eisenmenger syndrome. J Heart Lung Transplant 1996; 15: 100-105.

7 Diller GP, Gatzoulis MA. Pulmonary vascular disease in adults with congenital heart disease. Circulation 2007; 115: 1039-1050.

8 Levi DS, Scott V, Aboulhosn J. Pulmonary arterial hyperension in congenital heart disease. In: Humbert M, Lynch III JP, eds. Pulmonary Hypertension. London, Informa Healthcare UK, 2009; pp. 176-195.

9 Galiè N, Manes A, Palazzini M, et al. Management of pulmonary arterial hypertension associated with congenital systemic-topulmonary shunts and Eisenmenger syndrome. Drugs 2008; 68: 1049-1066.

10 Vogel M, Berger F, Kramer A, et al. Incidence of secondary pulmonary hypertension in adults with atrial septal or sinus venosus defects. Heart 1999; 82: 30-33.

11 Kidd L, Driscoll DJ, Gersony WM, et al. Second natural history study of congenital heart defects. Circulation 1993; 87: Suppl. 1, I-38-I-51.

12 Vongpatanasin W, Brickner ME, Hillis LD, et al. The Eisenmenger syndrome in adults. Ann Intern Med 1998; 128: 745-755.

13 Diller GP, Dimopoulos K, Broberg CS, et al. Presentation, survival prospects, and predictors of death in Eisenmenger syndrome: a combined retrospective and case-control study. Eur Heart J 2006; 27: 1737-1742.

14 Barst RJ, Foreman AJ, Ivy DD, et al. REVEAL Registry: one year outcome of patients with congenital heart disease-associated pulmonary arterial hypertension (CHD-APAH). Am J Respir Crit Care Med 2009; 179: A2656.

15 Dimopoulos K, Inuzuka SG, Giannakoulas G, et al. Improved survival among patients with Eisenmenger syndrome receiving advanced therapy for pulmonary hypertension. Circulation 2010; 121: $20-25$.

16 Duffels MG, van der Plas MN, Surie S, et al. Bosentan in pulmonary arterial hypertension: a comparison between congenital heart disease and chronic pulmonary embolism. Neth Heart $J$ 2009; 17: 334-338.

17 Duffels MG, Vis JC, van Loon RL, et al. Effect of bosentan on exercise capacity and quality of life in adults with pulmonary arterial hypertension associated with congenital heart disease with and without Down's syndrome. Am J Cardiol 2009; 103: 1309-1315.

18 Duffels MG, Vis JC, van Loon RL, et al. Down patients with Eisenmenger syndrome: is bosentan an option? Int J Cardiol 2009; 134: 378-383.

19 Van Loon RL, Hoendemis ES, Duffels MG. Long-term effect of bosentan in adults versus children with pulmonary arterial hypertension associated with systemic-to-pulmonary shunt: does the beneficial effect persist? Am Heart J 2007; 154: 776-782.

20 Perloff JK. Pediatric congenital cardiac becomes a postoperative adult: the changing population of congenital heart disease. Circulation 1973; 47: 606-619.

21 Perloff JK. Congenital heart disease in adults: a new cardiovascular subspecialty. Circulation 1991; 84: 1881-1890. 
22 Marelli AJ, Mackie AS, Ionescu-Ittu R, et al. Congenital heart disease in the general population: changing prevalence and age distribution. Circulation 2007; 115: 163-172.

23 Dearani JA, Connolly HM, Martinez R, et al. Caring for adults with congenital cardiac disease: successes and challenges for 2007 and beyond. Cardiol Young 2007; 17: Suppl. 2, 87-96.

24 van der Velde ET, Vriend JW, Mannens MM, et al. CONCOR, an initiative towards a national registry and DNA-bank of patients with congenital heart disease in the Netherlands: rationale, design, and first results. Eur J Epidemiol 2005; 20: 549-557.

25 Engelfriet P, Boersma E, Oechslin E, et al. The spectrum of adult congenital heart disease in Europe: morbidity and mortality in a 5 year follow-up period. The Euro Heart Survey on adult congenital heart disease. Eur Heart J 2005; 26: 2325-2333.

26 Gatzoulis MA, Alonso-Gonzalez R, Beghetti M. Pulmonary arterial hypertension in paediatric and adult patients with congenital heart disease. Eur Respir Rev 2009; 18: 154-161.
27 Engelfriet PM, Duffels MG, Möller T, et al. Pulmonary arterial hypertension in adults born with a heart septal defect: the Euro Heart Survey on adult congenital heart disease. Heart 2007; 93: 682-687.

28 Weijerman ME, van Furth $\mathrm{AM}$, van der Mooren MD, et al. Prevalence of congenital heart defects and persistent pulmonary hypertension of the neonate with Down syndrome. Eur J Pediatr 2010; 169: 1195-1199.

29 Malec E, Mroczek T, Pajak J, et al. Results of surgical treatment of congenital heart defects in children with Down's syndrome. Pediatr Cardiol 1999; 20: 351-354.

30 Suzuki K, Yamaki S, Mimori S, et al. Pulmonary vascular disease in Down's syndrome with complete atrioventricular septal defect. Am J Cardiol 2000; 86: 434-437.

31 Van de Bruaene A, Delcroix M, Pasquet A, et al. The Belgian Eisenmenger syndrome registry: implications for treatment strategies? Acta Cardiol 2009; 64: 447-453.

32 Vis JC, Thoonsen H, Duffels MG, et al. Six-minute walk test in patients with Down syndrome: validity and reproducibility. Arch Phys Med Rehabil 2009; 90: 1423-1427. 